\title{
Impact of Nanograting Phase-Shift on Light Absorption Enhancement in Plasmonics-Based Metal-Semiconductor-Metal Photodetectors
}

\author{
Narottam Das, ${ }^{1,2}$ Ayman Karar, ${ }^{1}$ Chee Leong Tan, ${ }^{3}$ Kamal Alameh, ${ }^{1,4}$ and Yong Tak Lee ${ }^{3,4,5}$ \\ ${ }^{1}$ Electron Science Research Institute, Edith Cowan University, Joondalup, WA 6027, Australia \\ ${ }^{2}$ Department of Electrical and Computer Engineering, Curtin University, Perth, WA 6845, Australia \\ ${ }^{3}$ School of Photonics Science, Gwangju Institute of Science and Technology (GIST), 261 Cheomdan-gwagiro (Oryong-dong), \\ Buk-gu, Gwangju 500-712, Republic of Korea \\ ${ }^{4}$ Department of Nanobio Materials and Electronics, Gwangju Institute of Science and Technology (GIST), \\ Gwangju 500-712, Republic of Korea \\ ${ }^{5}$ Department of Information and Communications, Gwangju Institute of Science and Technology (GIST), \\ Gwangju 500-712, Republic of Korea
}

Correspondence should be addressed to Narottam Das, narottam.das@curtin.edu.au and Kamal Alameh, k.alameh@ecu.edu.au

Received 3 March 2011; Accepted 10 May 2011

Academic Editor: Mikhail Vasiliev

Copyright ( 2011 Narottam Das et al. This is an open access article distributed under the Creative Commons Attribution License, which permits unrestricted use, distribution, and reproduction in any medium, provided the original work is properly cited.

The finite difference time-domain (FDTD) method is used to simulate the light absorption enhancement in a plasmonic metalsemiconductor-metal photodetector (MSM-PD) structure employing a metal nanograting with phase shifts. The metal fingers of the MSM-PDs are etched at appropriate depths to maximize light absorption through plasmonic effects into a subwavelength aperture. We also analyse the nano-grating phase shift and groove profiles obtained typically in our experiments using focused ion beam milling and atomic force microscopy and discuss the dependency of light absorption enhancement on the nano-gratings phase shift and groove profiles inscribed into MSM-PDs. Our simulation results show that the nano-grating phase shift blue-shifts the wavelength at which the light absorption enhancement is maximum, and that the combined effects of the nano-grating groove shape and phase shift degrade the light absorption enhancement by up to $50 \%$.

\section{Introduction}

In recent years, sub-wavelength nanostructured metal nanogratings have been identified as promising candidates for realising high-speed improved sensitivity metal-semiconductor-metal photodetectors (MSM-PDs) [1]. The strong interaction of a nanostructured metal grating with an incident light enables trapping the light through the metal finger slit into the semiconductor substrate, leading to substantial improvement in light absorption, and opening the way for a wide range of potential applications, such as optical fiber communications, high-speed chip-to-chip interconnects, and high-speed sampling $[2,3]$.

An MSM-PD is simply composed of two back-toback Schottky diodes and has interdigitated metal fingers deposited onto a semiconductor substrate as an active light absorption layer. Upon detection of photons, it collects the electric currents generated by photo-excited charge carriers in the semiconductor region, which drift under the electric field applied between the metal fingers. The speed of MSMPDs can be limited intrinsically by the carrier transit time between the electrodes. The interdigitated electrodes in MSM-PDs result in a huge increase in bandwidth and reduction in dark current, in comparison to conventional PIN photodiodes with active areas of similar size [2-4]. Recently, the use of surface plasmon-assisted effects has been reported for the development of MSM-PDs with a highresponsivity bandwidth product $[5,6]$.

Several theoretical and experimental results have been reported on the extraordinary optical transmission through the sub-wavelength metallic apertures, and metal gratings [7-20]. Based on finite-difference time-domain (FDTD) 


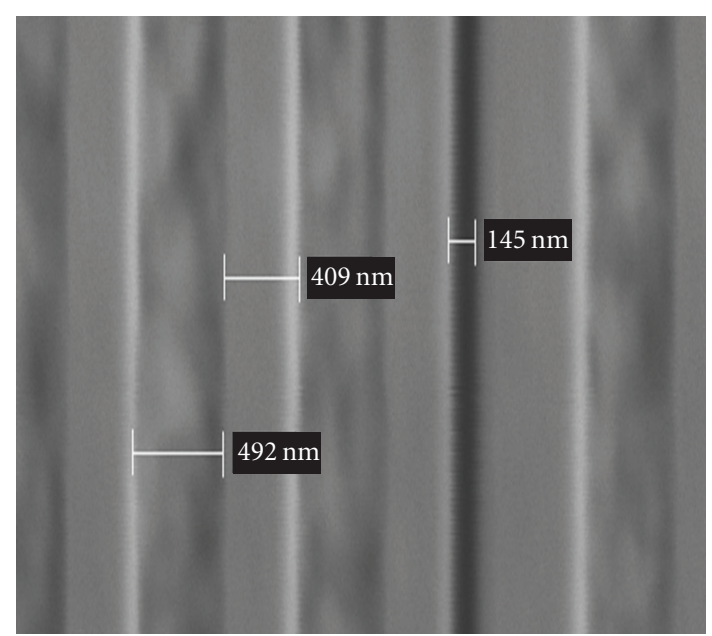

(a)

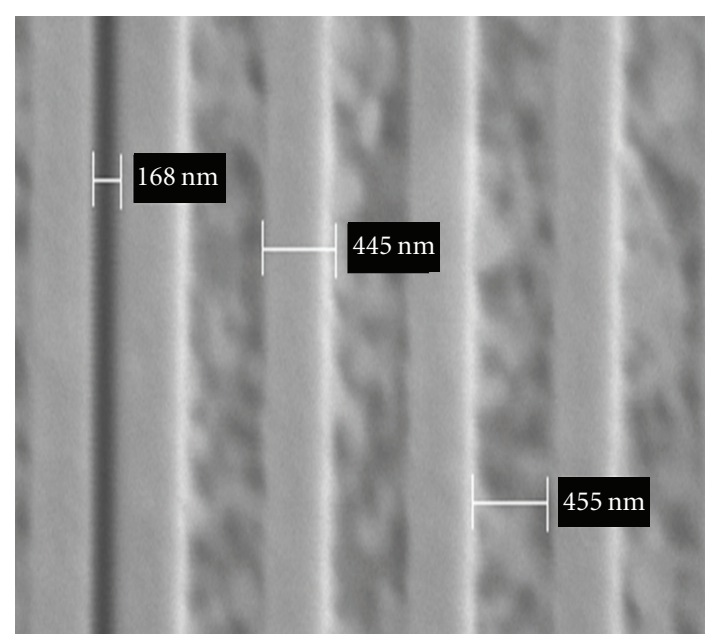

(b)

FIGURE 1: Typical SEM images of the metal nanogratings fabricated with FIB milling process for an estimated (predesigned/selected) subwavelength width of $100 \mathrm{~nm}$. (a) Nanograting phase shift is $(145-100) \mathrm{nm}=45 \mathrm{~nm}\left(\sim 20^{\circ}\right)$ and (b) nanograting phase shift is (168-100) nm $=68 \mathrm{~nm}\left(\sim 31^{\circ}\right)$.

simulation, results have shown significant enhancement of light absorption through interaction with surface plasmon polaritons (SPPs) for the design of MSM-PDs [1, 5-11].

In this paper, the FDTD method is used to simulate the light absorption enhancement in a GaAs MSM-PD structure, wherein the metal fingers are structured to form a metal nanograting above an unperturbed part of the metal fingers. The cross-sectional profiles of nanograting-assisted MSMPD samples patterned by focused ion beam (FIB) milling and the surface topography of the device are experimentally investigated using atomic force microscopy (AFM). Experimental results show that the real nanograting profile shapes are not rectangular (which would represent an ideal profile in terms of the achievable absorption enhancement) for grooves formed aiming at achieving a rectangular-shaped profile, but rather close to trapezoidal. We have previously investigated the effects of metal nanograting groove shapes [11] and analyzed their impact (or influence) on the light absorption enhancement in MSM-PDs. In this report, we carry out FDTD simulation on metal nanogratings with phase shifts [7] and show that, due to the combined effects of the nanograting groove shapes and the nanograting phase shift, the light absorption enhancement decreases and the wavelength, at which the light absorption enhancement is maximum, is red shifted.

\section{Plasmonics-Based MSM-PD Srtucture Design}

2.1. Experimental Observation of Nanograting Phase Shift in MSM-PD. In this section, we discuss the experimental observation of nanograting phase shift of slits (aperture widths) in MSM-PD structures. Figure 1 shows the SEM images of two MSM-PD nanogratings etched inside the top part of the gold ( $\mathrm{Au}$ ) layer using the FIB milling system. A $10 \mathrm{~nm} \mathrm{Cr}$ adhesion layer and a $190 \mathrm{~nm}$-thick Au layer were deposited on top of the GaAs substrate using an e-beam evaporator to form the Schottky-diode pads (cathode and anode). FIB lithography (which has superior performance for cutting sharp-edge profiles compared to conventional photolithography systems) was used to partially etch the Au layer and fully etch a $100 \mathrm{~nm}$ aperture, as shown in Figure 1. The FIB-etched aperture widths were $145 \mathrm{~nm}$ and $168 \mathrm{~nm}$, respectively, causing nanograting phase shifts of $(145-100) \mathrm{nm}=45 \mathrm{~nm}\left(\sim 20^{\circ}\right)$, and $(168-100) \mathrm{nm}=68 \mathrm{~nm}$ $\left(\sim 31^{\circ}\right)$ as shown in Figures $1(\mathrm{a})$ and $1(\mathrm{~b})$, respectively. The term "nanograting" phase shift $(\delta)$ is defined as, $\delta \equiv$ $2 \pi x_{g p} / \Lambda$, where, $x_{g p}$ is half of the difference between the widths of the first metal ridges of the nanograting, and $\Lambda$ is the metal nanograting period (illustrated in Figure 4). It was also observed, through SEM imaging, that the nanograting profile is trapezoidal (or like a V-grooved shape), rather than rectangular. This trapezoidal shape results from the redeposition of gold atoms and truncation of nanograting edges by the etching ion beam.

In addition to the SEM images (shown in Figure 1), and to further confirm the nanograting groove shapes, an AFM system (XE-100 from Park system) was used to analyze the surface topography of the nanogratings. The AFM images shown in Figures 2(a) and 2(b) confirmed the shape of the nanograting is nearly trapezoidal profiles and the aspect ratios are between 0.5 and 0.8 (approximate measurement). The aspect ratio is defined as the trapezoid top-to-bottom base ratio.

Based on these experimental results (SEM and AFM images), the groove shape of the nanograting was taken into account in conjunction with the phase shift of the nanogratings to simulate the light absorption enhancement in plasmonics-based MSM-PDs.

2.2. Modelling of Plasmonics-Based MSM-PDs. In this section, we discuss the design of plasmonics-based high-speed MSM-PD structures having different types of nanograting 
Line profile: red

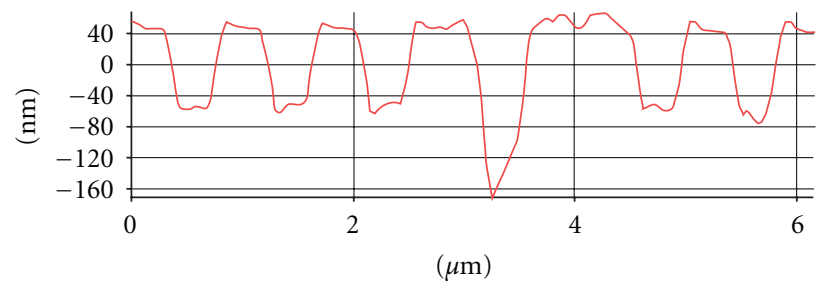

(a)
Line profile: red

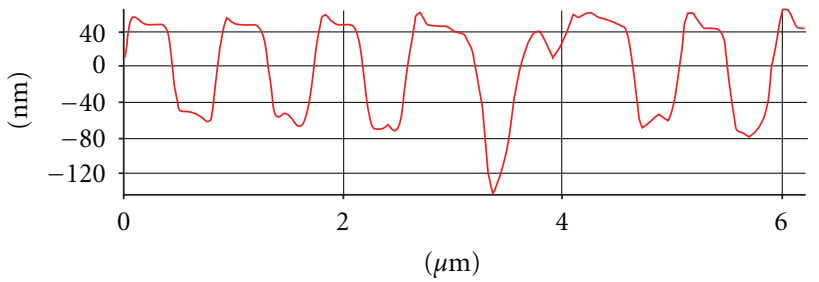

(b)

FIGURE 2: AFM images of two MSM-PD structures having different nanograting profiles by FIB milling.

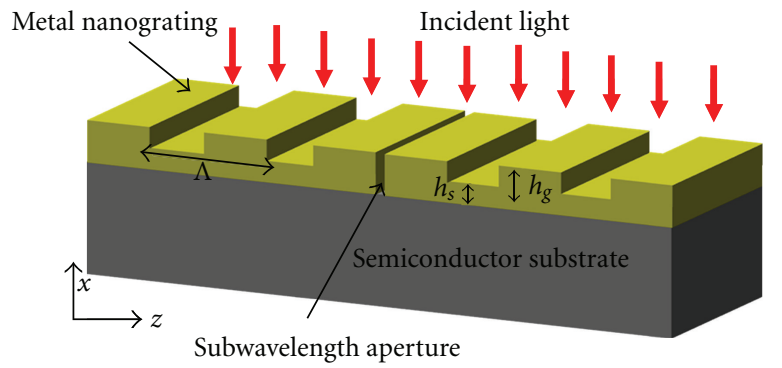

FIGURE 3: Schematic diagram of a typical MSM-PD structure with plasmonic nanogratings etched inside the top part of the Au layer. The metal nanograting profile is rectangular shaped.

groove profiles and nanograting phase shifts. Figure 3 illustrates a plasmonic-based MSM-PD structure, which consists of three separate layers, namely, (i) a top layer (metal nanograting), (ii) an unperturbed metal layer (underlayer) containing conventional sub-wavelength apertures, and (iii) a semiconductor (GaAs) substrate. For a metal nanograting with the period of $\Lambda$, the wave vector of the excited SPPs $\left(k_{s p}\right)$ is given by $[1,8-11]$

$$
k_{s p}=\frac{\omega}{c} \sin \theta \pm \frac{2 \pi l}{\Lambda}=\frac{\omega}{c} \sqrt{\frac{\varepsilon_{m}^{\prime} \varepsilon_{d}}{\varepsilon_{m}^{\prime}+\varepsilon_{d}}}
$$

where $\omega$ is the angular frequency of the incident light wave, $c$ is the speed of light in vacuum, and $\theta$ is its angle of incidence with respect to the device normal, and $l$ is an integer number. In this analysis, we denote the complex dielectric permittivity of the metal as $\varepsilon_{m}=\varepsilon_{m}^{\prime}+i \varepsilon_{m}^{\prime \prime}$ and the dielectric permittivity of air is denoted as $\varepsilon_{d}$.

Using (1), a period of $\Lambda=810 \mathrm{~nm}$ for the Au/air interface corresponds to a GaAs MSM-PD with an edge of absorption of around $830 \mathrm{~nm}$ for $\theta=0$. We selected the duty cycle of grating corrugations to be $50 \%$. A typical MSM-PD structure with a rectangular Au nanograting is shown in Figure 3. The dependency of the plasmon-assisted light absorption enhancement on the geometric shape of the nanograting corrugations cross-sections and grating phase shift was investigated using the FDTD simulation method. In our simulation, the thickness of the unperturbed metal layer containing sub-wavelength apertures shown as $h_{s}$ was $10 \mathrm{~nm}$ and the height of the metallic nanogratings, $h_{g}$, was $90 \mathrm{~nm}$.

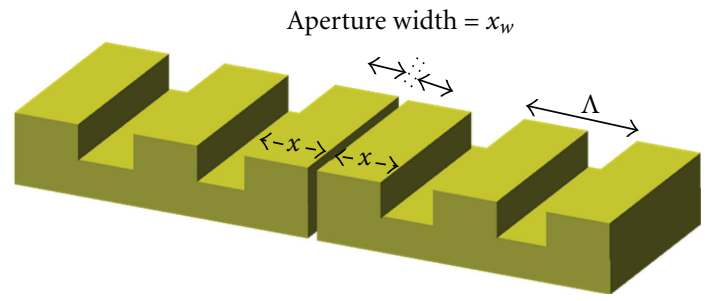

(a)

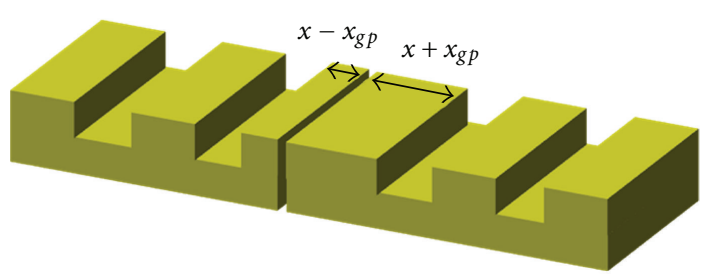

(b)

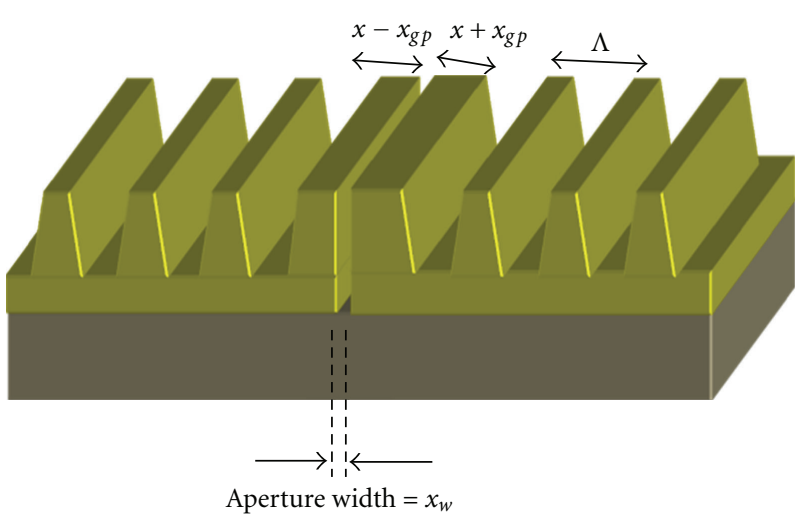

(c)

FIGURE 4: Schematic diagrams of different Au nanogratings (a) without the phase shift and (b) with the phase shift, $\delta \equiv 2 \pi x_{g p} / \Lambda$. (c) General trapezoidal-shaped nanograting profile, with the aspect ratio defined as the trapezoid top-to-bottom base ratio.

The sub-wavelength aperture width $x_{w}$ was varied between 50 to $200 \mathrm{~nm}$.

Figure 4 shows schematic diagrams of the nanograting without (a) and with (b) a phase shift defined as $\delta \equiv$ $2 \pi x_{g p} / \Lambda$, respectively, where $x_{g p}$ is half of the difference 


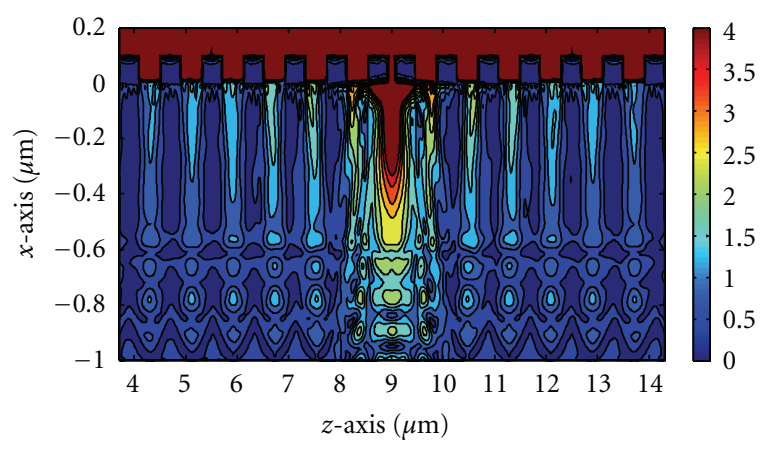

(a)

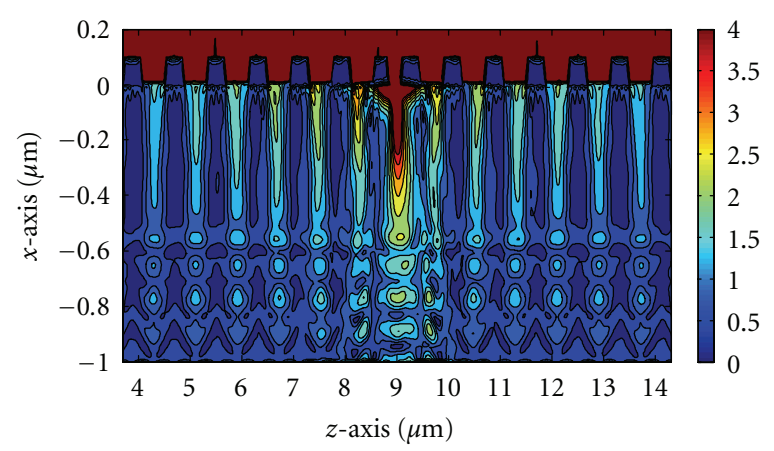

(b)

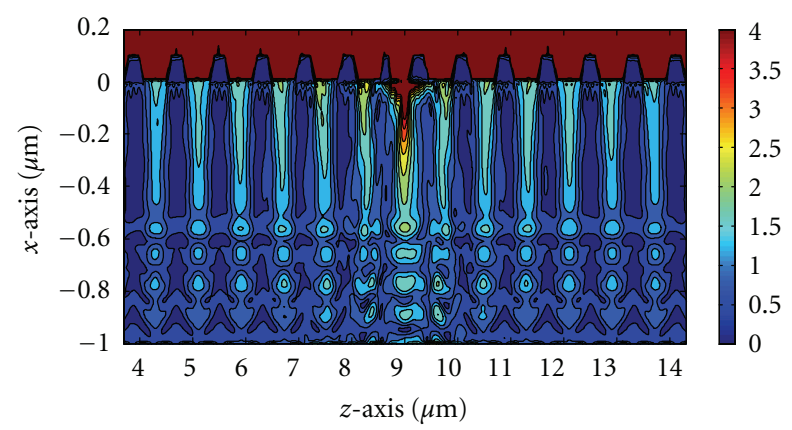

(c)

FIGURE 5: Total electric field intensity distribution in the cross-section of computational volume behind the nanograting with different grooves cross-section and the following grating parameters: the nanograting height is $90 \mathrm{~nm}$, unperturbed gold (Au) layer thickness is $10 \mathrm{~nm}$, grating period is $810 \mathrm{~nm}$, and the sub-wavelength aperture width is $100 \mathrm{~nm}$. The color scale has been optimized for representing small weak-field intensity variations. The nanograting profiles are (a) rectangular shaped (aspect ratio 1 ) with the grating phase shift $0^{\circ}$, (b) trapezoidal shaped (with an aspect ratio of 0.8 ) with a grating phase shift of $\sim 45^{\circ}$, and (c) trapezoidal shaped (with an aspect ratio of 0.5 ) with a grating phase shift of $\sim 90^{\circ}$.

between the widths of the first metal ridges of the nanograting, and $\Lambda$ is the metal nanograting period.

The general trapezoidal-shaped nanograting profile was considered more practical than the ideal rectangular-shaped profile, because it is the closest cross-sectional profile to that developed using FIB milling in the case of small-featuresize (sub-100 nm) nanopatterned grooves. Two trapezoidalshaped gratings were simulated, which had aspect ratios (trapezoid-top to base length) of 0.8 and 0.5 , respectively. Figure 4(c) illustrates a trapezoidal-shaped nanograting profile with grating phase shift. In the simulation, the grating phase was varied from 0 to around $90^{\circ}$.

The incident light normally passes through the subwavelength apertures and reaches the GaAs substrate, thus generating electron-hole pairs. In addition, the light absorption within GaAs is assisted by the SPPs excited near the metal-semiconductor interface near the nanograting region. The energy of light incident on the metal nanograting is partially coupled into propagating SPPs that increase the light coupled into the sub-wavelength aperture, thus improving the light absorption efficiency in the semiconductor substrate. The improvement in light absorption is due to SPP-generated localized regions of high-intensity electric field distribution (as illustrated in Figure 5). Therefore, the nanograting acts as a light concentrator (plasmonic lens) or collector triggering extraordinary optical transmission (and therefore absorption) within the active regions of the photodetector.

MSM-PD designs of different groove shapes (with and without the metal nanograting) were simulated using the Opti-FDTD software package developed by Optiwave Inc. In the simulation, we used a mesh step size of $\Delta x=10 \mathrm{~nm}$ and a time step of $\Delta t<0.1 \Delta x / c$. The excitation field was modeled as a Gaussian-modulated continuous wave. The incident light wave was TM-polarized (its electric field oscillation direction was along the $z$-axis, perpendicular to the nanograting grooves). The anisotropic perfectly matched layer (APML) boundary conditions were applied in both $x$ and $z$-directions. The gold $\mathrm{(Au})$ dielectric permittivity was defined by the Lorentz-Drude model [21] and the dielectric permittivity data of GaAs was taken from [22].

A density plot of the total (transmitted) electric field strength within the substrate cross-section is shown in Figure 5. We used a custom-designed Matlab algorithm to calculate the total electric field intensity distribution, which results from the vector summation of the modeled complex electric field component distributions along the $x$-direction $\left(E_{x}\right)$ and the $z$-direction $\left(E_{z}\right)$. From Figure 5 , it is clearly seen that the significant amount of light passing through the region of sub-wavelength aperture width is mainly due 


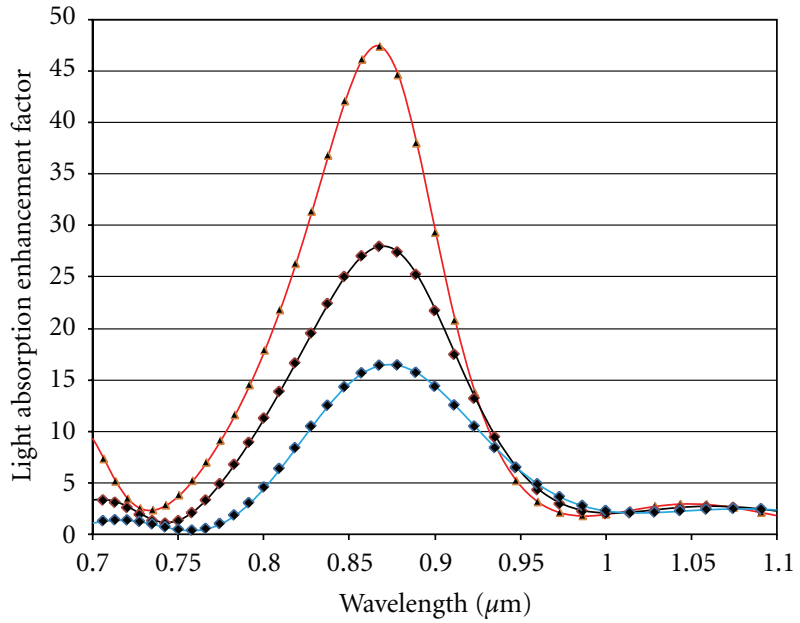

Subwavelength aperture width

$\leftarrow 50 \mathrm{~nm}$

$\rightarrow-100 \mathrm{~nm}$

- $200 \mathrm{~nm}$

FIGURE 6: Light absorption enhancement factor spectra for MSMPDs with plasmon-assisted operation for different sub-wavelength aperture widths: such as, $50 \mathrm{~nm}, 100 \mathrm{~nm}$, and $200 \mathrm{~nm}$. For this case, the grooves shape was rectangular.

to the plasmon-assisted effects, which result in propagating SPPs excited by the incident light waves and concentrated (by plasmonic lenses) within the photodetector's active region. The light transmission inside the substrate depends on the aperture size and the nanograting profile. Figure $5(\mathrm{a})$ shows the total electric field intensity distribution (generated using the computed complex electric field distribution) inside the substrate cross-section for an ideal rectangular-shaped nanograting with the grating phase shift $0^{\circ}$. Figures $5(\mathrm{~b})$ and 5(c) show the total electric field intensity distribution inside the substrate for more practical trapezoidal-shaped (aspect ratio of 0.8 and 0.5 ) nanograting profiles with the nanograting phase shift of $45^{\circ}$ and $90^{\circ}$, respectively. It can be noted that the light transmission inside the substrate of the trapezoidal-shaped nanograting MSM-PD is lower than that of a rectangular-shaped nanograting MSM-PD. This reduction is due to the combined effects of the nanograting groove shapes and the nanograting phase shift, as discussed later.

\section{Results and Discussion}

In this section, we discuss the simulation results of different MSM-PD structures having different types of nanograting groove-geometry designs as well as the light absorption enhancement dependency on the sub-wavelength aperture widths and the nanograting phase shift. We particularly modeled the impact of the nanograting groove shape, the nanograting phase shift, and the aperture width on the light absorption enhancement. The light absorption enhancement factor is defined as the ratio of the normalized power transmittance of MSM-PD with the metal nanograting to the

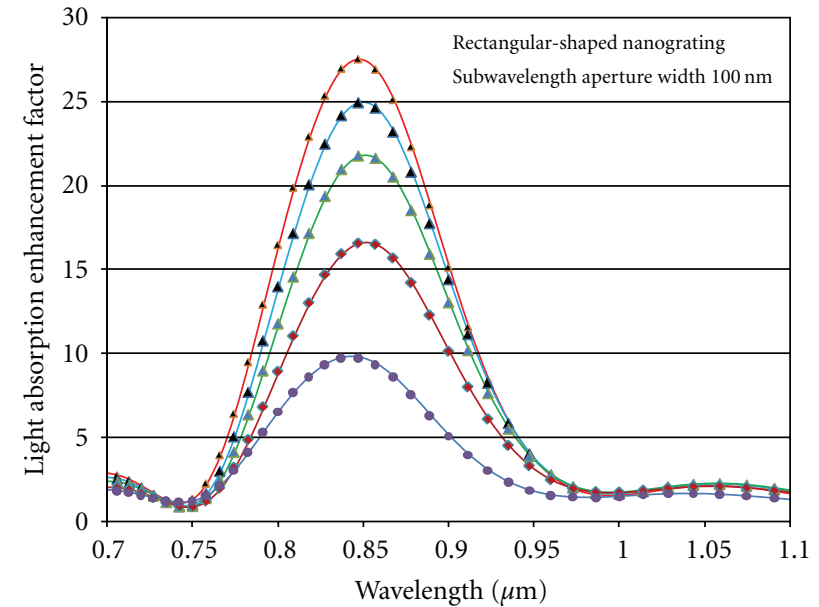

Nanograting phase shift

$$
\begin{aligned}
& \rightarrow 0^{\circ} \rightarrow 45^{\circ} \\
& \leftarrow 9^{\circ} \quad \longrightarrow 90^{\circ} \\
& \rightarrow 22.5^{\circ}
\end{aligned}
$$

FIGURE 7: Light absorption enhancement factor spectra for MSMPDs with plasmon-assisted operation for various grating phase shifts. Here, the nanograting phase shift is varied from $0^{\circ}$ to $90^{\circ}$. The sub-wavelength aperture width was kept constant at $100 \mathrm{~nm}$ and the groove shape was rectangular.

normalized power transmittance of the same device without a metal nanograting $[10,11]$.

Figure 6 shows the simulated light absorption enhancement factor spectra of MSM-PD structures with different aperture widths, namely, $50 \mathrm{~nm}, 100 \mathrm{~nm}$, and $200 \mathrm{~nm}$. The grooves of the nanograting were rectangular shaped in their cross-sections. The simulation results show that the light absorption enhancement factor decreases rapidly with the increasing of sub-wavelength aperture width. We obtained a light enhancement factor of about 50 times for a $50 \mathrm{~nm}$ wide sub-wavelength aperture width, about 28 times for a $100 \mathrm{~nm}$-wide sub-wavelength aperture and about 16 times for a $200 \mathrm{~nm}$-wide sub-wavelength aperture width.

From the above results (shown in Figure 6), the subwavelength aperture width of $100 \mathrm{~nm}$ can be realized with an FIB milling system. Figure 7 shows the simulated light absorption enhancement factor spectra of several optimized MSM-PD structures with different grating phase shifts (between $0^{\circ}$ and $90^{\circ}$ ). The results show that the light absorption enhancement factor decreases rapidly with increasing the grating phase shift. The light absorption enhancement factor is about 10 times for a grating phase shift of $0^{\circ}$. However, when the grating phase shift is increased to $90^{\circ}$ (for the same aperture width) the light absorption enhancement factor drops to below 10 times (as shown in Figure 7). Also note from Figure 7 that the peak wavelength position is shifted from $850 \mathrm{~nm}$ to $837 \mathrm{~nm}$ when the grating phase shift increased from $0^{\circ}$ to $90^{\circ}$.

Figures $8(\mathrm{a})$ and $8(\mathrm{~b})$ show the spectral distribution of the light absorption enhancement factor for trapezoidal nanograting groove shapes with aspect ratios of 0.8 and 0.5 , 


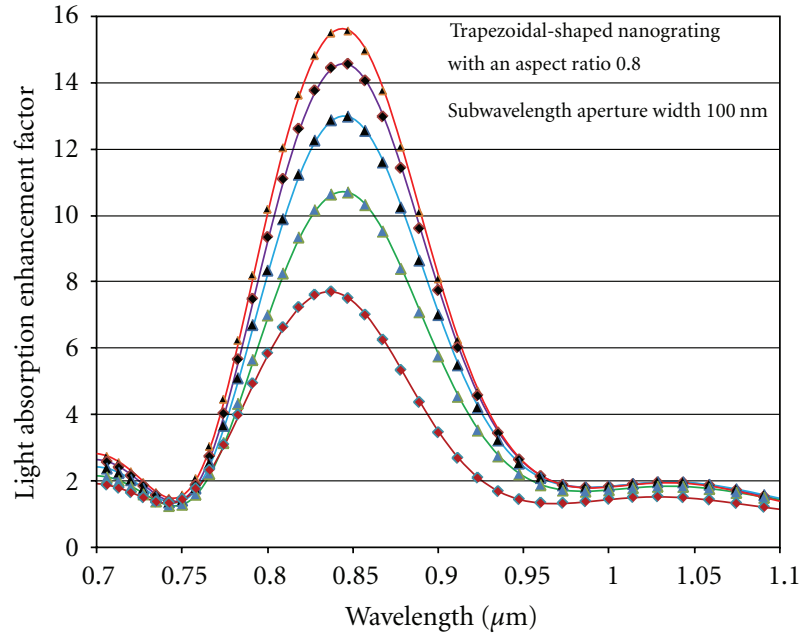

Nanograting phase shift $\begin{array}{ll}\leftarrow 0^{\circ} & \rightarrow 45^{\circ} \\ \rightarrow-9^{\circ} & \rightarrow-90^{\circ} \\ \leftarrow 22.5^{\circ} & \end{array}$

(a)
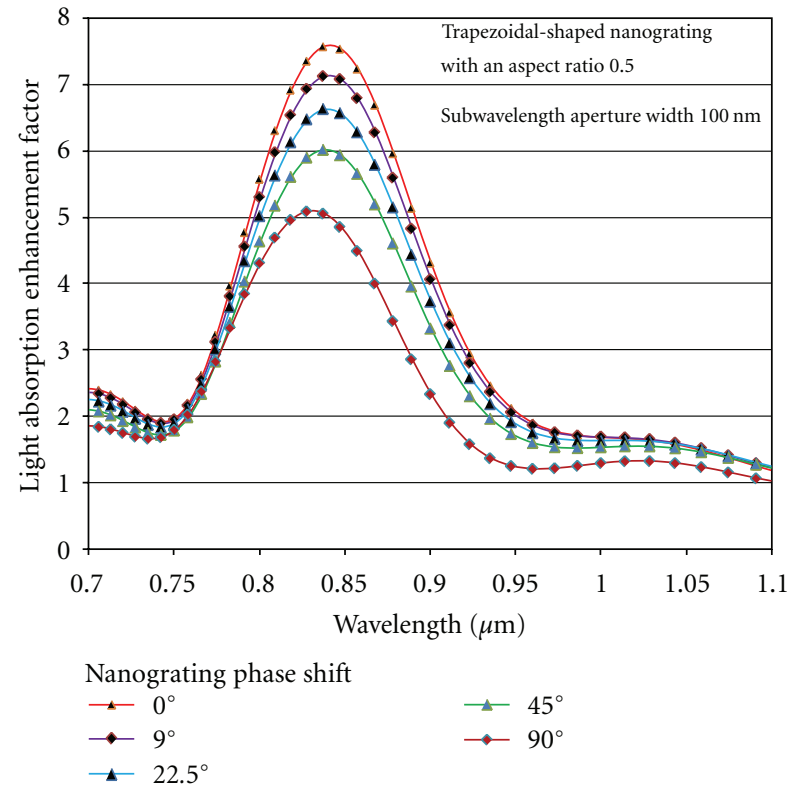

(b)

FIGURE 8: Light absorption enhancement factor spectra for trapezoidal-shaped nanograting with the aspect ratios of 0.8 (a) and 0.5 (b) for different nanograting phase shifts. In this case, the sub-wavelength aperture width was kept constant at $100 \mathrm{~nm}$.

respectively, for different grating phase shifts. From Figure 8 we notice that the light absorption enhancement factor decreases slowly with increasing the grating phase shift. However, for a smaller aspect ratio the light absorption enhancement factor is smaller. Also, when the grating phase shift is $90^{\circ}$ the degradation in light absorption enhancement drops by $50 \%$ in comparison to the case of a $0^{\circ}$ grating phase shift. Note that, from Figures $8(\mathrm{a})$ and $8(\mathrm{~b})$, the combined impact (or influence) of the groove shape and the grating phase shift is a reduction in the light absorption enhancement factor from 15 to around 5 .

The maximum light absorption enhancement factor versus the nanograting phase shift is shown in Figure 9, for rectangular and trapezoidal grating profiles. It is clear that the light absorption enhancement factor decreases rapidly with increasing the nanograting phase shift for rectangular shaped nanogratings. However, for trapezoidalshaped nanogratings, the rate at which the light absorption enhancement factor decreases is small in comparison with that of the rectangular-shaped nanograting profile.

Figure 10 shows the peak wavelength at maximum light absorption versus the nanograting phase shift characteristics for different nanograting profiles. In this simulation, we considered the constant grating period of $810 \mathrm{~nm}$ and the nanograting phase shifts were varied from $0^{\circ}$ to $90^{\circ}$ for a constant grating height of $90 \mathrm{~nm}$. For rectangular-shaped (when an aspect ratio is 1) nanograting profiles the maximum light absorption enhancement peak was about $847 \mathrm{~nm}$ and it was not affected with the nanograting phase shifts, that is, the peak wavelength was independent on the nanograting phase shift. However, for a trapezoidal-shaped nanograting profile with an aspect ratio of 0.8 , the peak wavelength shift drifted

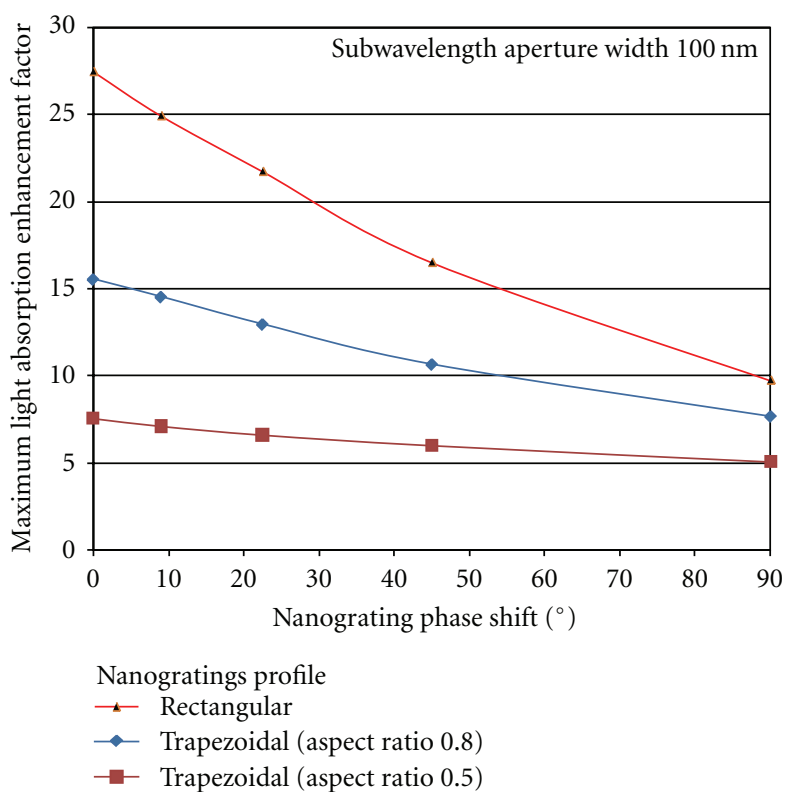

FIGURE 9: Maximum light absorption enhancement factor versus the grating phase shift for rectangular and trapezoidal (aspect ratios are 0.8 and 0.5 ) shaped nanograting geometries. In this case, the subwavelength aperture width was kept constant at $100 \mathrm{~nm}$.

from $847 \mathrm{~nm}$ to $837 \mathrm{~nm}$ when the nanograting phase shift was increased from $0^{\circ}$ to $90^{\circ}$. For the same conditions, the peak wavelength shift drifted from $837 \mathrm{~nm}$ to $827 \mathrm{~nm}$, when the aspect ratio was 0.5 for a trapezoidal-shaped nanograting profile. These variations, as well as the light absorptionenhancing performance differences between the different 


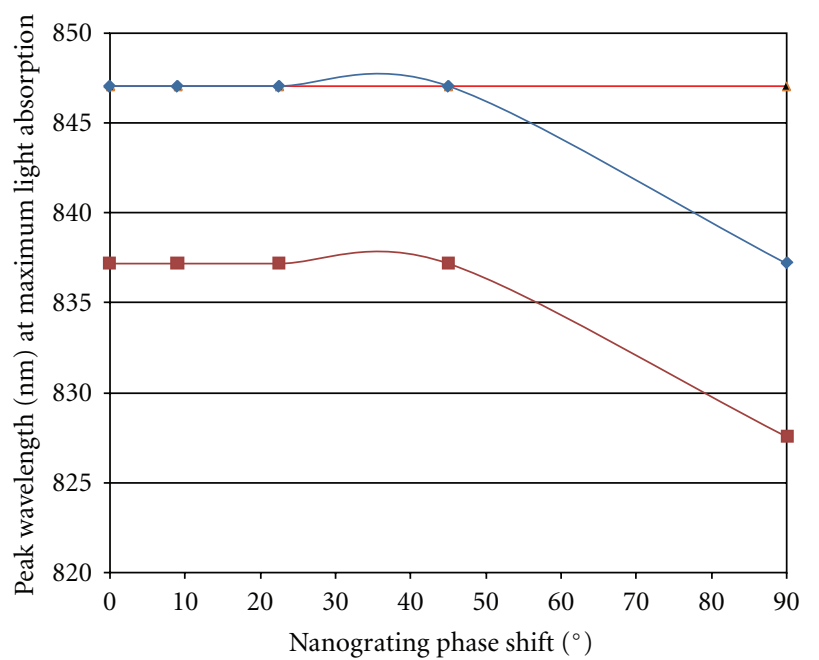

Nanogratings profile
$\neg$ Rectangular
$\longrightarrow$ Trapezoidal (aspect ratio 0.8)
$\rightarrow-$ Trapezoidal (aspect ratio 0.5)

FIgURE 10: Peak wavelength at maximum light absorption enhancement versus the nanograting phase shift characteristics for different nanogratings profile, where the grating height is $90 \mathrm{~nm}$. Here, the nanograting profiles are rectangular and trapezoidal (aspect ratio 0.8 and 0.5$)$ shaped.

nanograting profiles are likely due to the shape-dependent and phase shift differences in the effective refractive indices of the nanopatterned metal layers.

\section{Conclusions}

We have modeled the light-capture performance of MSMPD structures employing metal nanogratings of different cross-sectional profiles in conjunction with the nanograting phase shift in terms of the light absorption enhancement and hence the responsivity-bandwidth product. The FDTD technique has been used to optimize the different device parameters, such as sub-wavelength aperture width, nanograting shapes, grating phase shift, and other structure parameters for maximized the light absorption enhancement. Our simulation results have shown that an optimsed MSM-PD structure can attain a maximum light absorption enhancement of about 28 times (with $100 \mathrm{~nm}$-wide sub-wavelength aperture width) better than conventional MSM-PDs. Furthermore, the simulation results of practical trapezoidal-shaped nanogratings have shown that for a $90^{\circ}$ phase shift, the light absorption enhancement drops by around $50 \%$. These results are useful for the design and development of plasmonics-based high-speed MSM-PDs.

\section{Acknowledgments}

The authors acknowledge the support of the Faculty of Computing, Health and Science, Edith Cowan University, Australia, and the Department of Nano-bio Materials and
Electronics, Gwangju Institute of Science and Technology, Republic of Korea.

\section{References}

[1] J. A. Shackleford, R. Grote, M. Currie, J. E. Spanier, and B. Nabet, "Integrated plasmonic lens photodetector," Applied Physics Letters, vol. 94, no. 8, Article ID 083501, 2009.

[2] J. B. D. Soole and H. Schumacher, "InGaAs metalsemiconductor-metal photodetectors for long wavelength optical communications," IEEE Journal of Quantum Electronics, vol. 27, no. 3, pp. 737-752, 1991.

[3] M. Ito and O. Wada, "Low dark current GaAs metalsemiconductor-metal (MSM) photodiodes using WSix contacts," IEEE Journal of Quantum Electronics, vol. 22, no. 7, pp. 1073-1077, 1986.

[4] L.-H. Laih, T.-C. Chang, Y.-A. Chen, W.-C. Tsay, and J.-W. Hong, "Characteristics of MSM photodetectors with trench electrodes on P-type Si wafer," IEEE Transactions on Electron Devices, vol. 45, no. 9, pp. 2018-2023, 1998.

[5] J. Hetterich, G. Bastian, N. A. Gippius, S. G. Tikhodeev, G. von Plessen, and U. Lemmer, "Optimized design of plasmonic MSM photodetector," IEEE Journal of Quantum Electronics, vol. 43, no. 10, pp. 855-859, 2007.

[6] S. Collin, F. Pardo, R. Teissier, and J.-L. Pelouard, "Efficient light absorption in metal-semiconductor-metal nanostructures," Applied Physics Letters, vol. 85, no. 2, pp. 194-196, 2004.

[7] R. D. R. Bhat, N. C. Panoiu, R. M. Osgood Jr., and S. R. J. Brueck, "Enhancing infrared photodetection with a circular metal grating," in Conference on Lasers and Electro-Optics (CLEO '07), Baltimore, Md, USA, May 2007.

[8] H. Raether, Surface Plasmons on Smooth, Rough Surfaces, and Gratings, Springer, Berlin, Germany, 1988.

[9] S. A. Maier, Plasmonics: Fundamentals and Applications, Springer Science, New York, NY, USA, 2007.

[10] C. L. Tan, V. V. Lysak, K. Alameh, and Y. T. Lee, "Absorption enhancement of $980 \mathrm{~nm}$ MSM photodetector with a plasmonic grating structure," Optics Communications, vol. 283, no. 9, pp. 1763-1767, 2010.

[11] N. Das, A. Karar, M. Vasiliev, C. L. Tan, K. Alameh, and Y. T. Lee, "Analysis of nano-grating-assisted light absorption enhancement in metal-semiconductor-metal photodetectors patterned using focused ion-beam lithography," Optics Communications, vol. 284, no. 6, pp. 1694-1700, 2011.

[12] F. J. García-Vidal and L. Martín-Moreno, "Transmission and focusing of light in one-dimensional periodically nanostructured metals," Physical Review B, vol. 66, no. 15, Article ID 155412, 2002.

[13] G. Lévêque, O. J. F. Martin, and J. Weiner, "Transient behavior of surface plasmon polaritons scattered at a subwavelength groove," Physical Review B, vol. 76, no. 15, Article ID 155418, 2007.

[14] B. Sturman, E. Podivilov, and M. Gorkunov, "Theory of extraordinary light transmission through arrays of subwavelength slits," Physical Review B, vol. 77, no. 7, Article ID 075106, 2008.

[15] T. W. Ebbesen, H. J. Lezec, H. F. Ghaemi, T. Thio, and P. A. Wolff, "Extraordinary optical transmission through subwavelength hole arrays," Nature, vol. 391, no. 6668, pp. 667$669,1998$.

[16] L. Martín-Moreno, F. J. García-Vidal, H. J. Lezec et al., "Theory of extraordinary optical transmission through subwavelength hole arrays," Physical Review Letters, vol. 86, no. 6, pp. 1114-1117, 2001. 
[17] F. J. García-Vidal, H. J. Lezec, T. W. Ebbesen, and L. MartínMoreno, "Multiple paths to enhance optical transmission through a single subwavelength slit," Physical Review Letters, vol. 90, no. 21, Article ID 213901, 2003.

[18] L. Martín-Moreno, F. J. Garcia-Vidal, H. J. Lezec, A. Degiron, and T. W. Ebbesen, "Theory of highly directional emission from a single subwavelength aperture surrounded by surface corrugations," Physical Review Letters, vol. 90, no. 16, Article ID 167401, 4 pages, 2003.

[19] H. J. Lezec and T. Thio, "Diffracted evanescent wave model for enhanced and suppressed optical transmission through subwavelength hole arrays," Optics Express, vol. 12, no. 16, pp. 3629-3651, 2004.

[20] J. S. White, G. Veronis, Z. Yu et al., "Extraordinary optical absorption through subwavelength slits," Optics Letters, vol. 34, no. 5, pp. 686-688, 2009.

[21] A. D. Rakić, A. B. Djurišić, J. M. Elazar, and M. L. Majewski, "Optical properties of metallic films for vertical-cavity optoelectronic devices," Applied Optics, vol. 37, no. 22, pp. 52715283, 1998.

[22] E. D. Palik, "Galllium Arsenide (GaAs)," in Handbook of Optical Constants of Solids, E. D. Palik, Ed., Academic Press, San Diego, Calif, USA, 1985. 

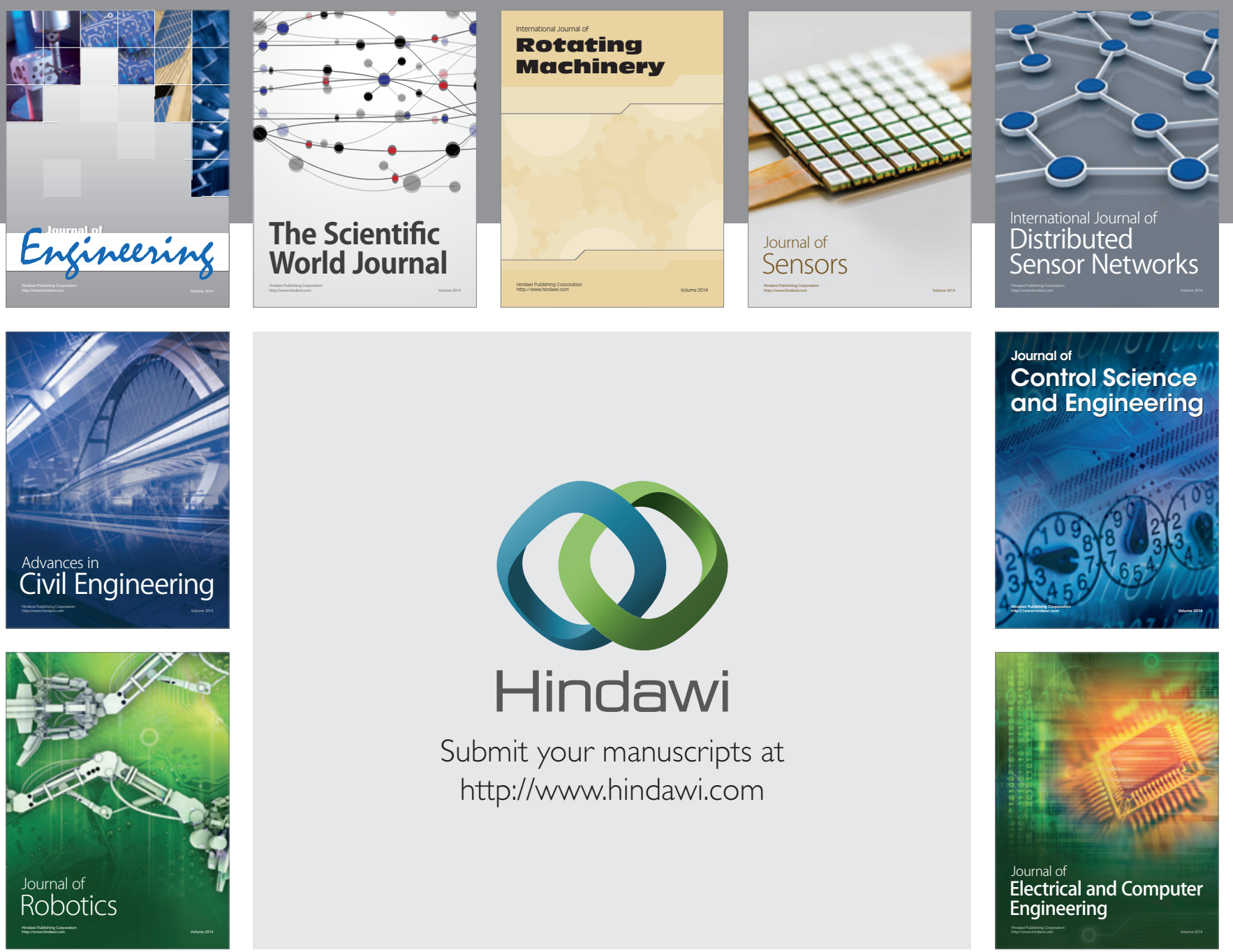

Submit your manuscripts at

http://www.hindawi.com
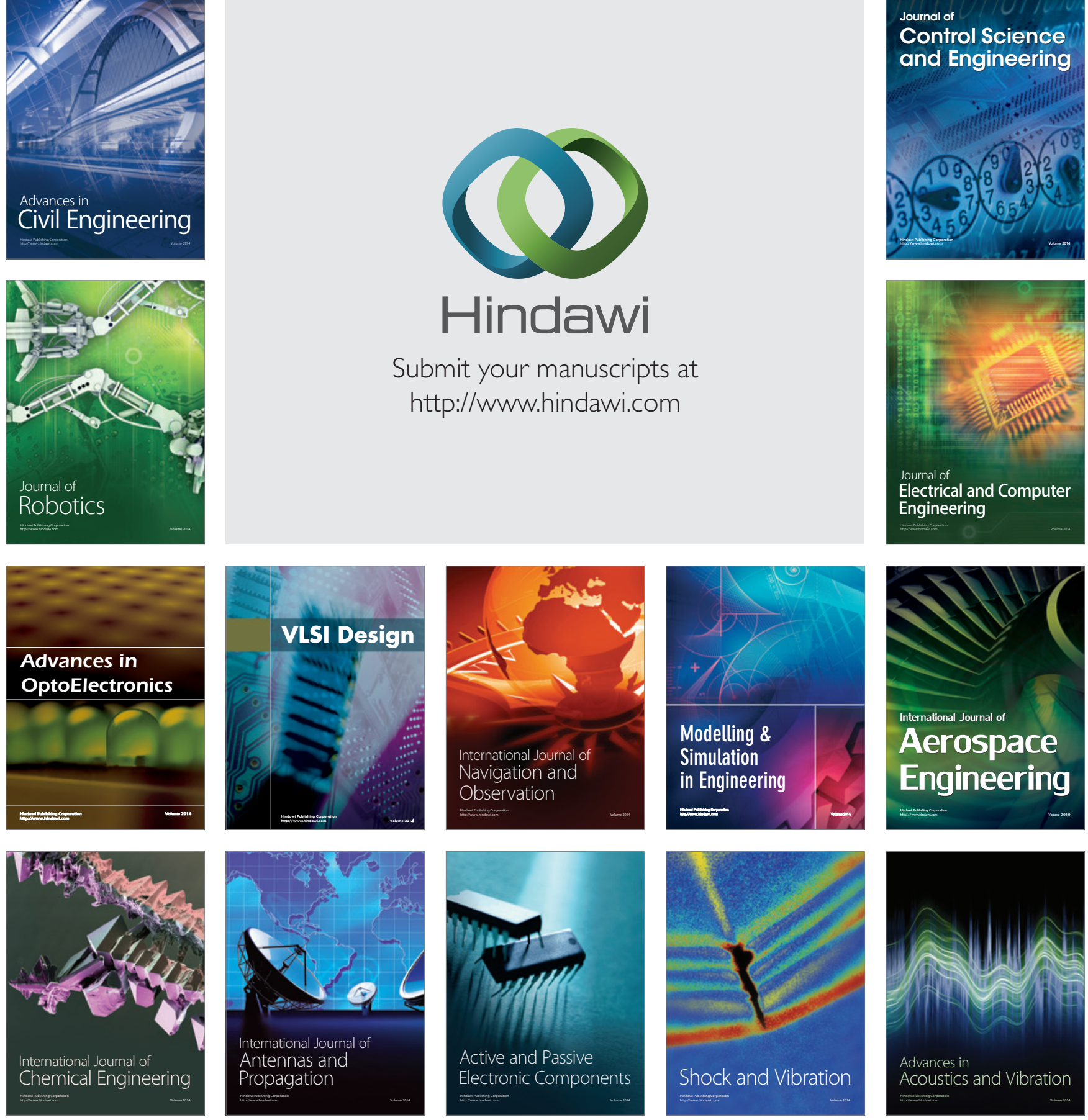